\title{
Multidisciplinary team management in thoracic oncology: more than just a concept?
}

\author{
Helen A. Powell'1 David R. Baldwin² \\ Number 1 in the series "Challenges and controversies in thoracic oncology" \\ Edited by J-P. Sculier, B. Besse and P. Van Schil \\ Affiliations: \\ 'Nottingham Respiratory Research Unit, University of Nottingham, Nottingham, UK. \\ ${ }^{2}$ Respiratory Medicine, Nottingham University Hospitals NHS Trust, City Hospital Campus, Nottingham, UK.
}

\section{Correspondence:}

D.R. Baldwin, Respiratory Medicine Unit, David Evans Research Centre, Nottingham City Hospital, Hucknall Road, Nottingham, NG5 1PB, UK.

E-mail: david.baldwinanuh.nhs.uk

ABSTRACT Multidisciplinary team (MDT) management in thoracic oncology has been introduced over the past two decades with the aim of improving outcomes for patients. While MDT management has become the standard of care in some countries, not all healthcare systems have adopted this practice. In this article we review the history and implementation of MDT care in thoracic oncology and explore the evidence for, and challenges associated with, this system of working.

There are many advantages of an MDT both to the patient, the clinicians and the wider population, but it is difficult to demonstrate a beneficial effect on outcomes such as treatment rates or survival given the substantial number of coexistent changes in the management of thoracic malignancies over the same time period. There are also some disadvantages associated with MDT working, particularly the costs of setting up the service and the time commitment from each of the healthcare professionals involved. Barriers to effective MDT working include poor attendance by some specialists, inadequate preparation and poor quality information about the patient. Variation in quality of MDTs has been reported so it is important that practice is monitored and areas for improvement identified.

@ERSpublications

Multidisciplinary teams ensure patients receive timely specialist input but effect on treatment/ survival is hard to show http://ow.ly/sC7dL

For editorial comments see page 1571.

Received: Aug 302013 | Accepted after revision: Dec 142013 | First published online: Feb 132014

Conflict of interest: Disclosures can be found alongside the online version of this article at www.erj.ersjournals.com

Copyright @ERS 2014 


\section{Introduction}

The pathway of care for patients with suspected thoracic malignancy varies considerably in different healthcare systems. This is evident from the very start of the pathway, where some patients are referred through a primary care doctor while others refer themselves directly to a pulmonologist, oncologist, thoracic surgeon or even a general surgeon. However, in some countries, in an effort to standardise care, it is now standard practice for all patients who have cancer, including those with thoracic malignancies, to be referred to and be managed by a cancer site-specific multidisciplinary team (MDT). This facilitates the consensus opinion of all of the relevant specialists and other healthcare professionals, before a management plan is decided.

In this article we review the history and implementation of MDT care in thoracic oncology and explore the evidence for, and challenges associated with, this system of working. Our personal experience is predominantly of MDT management of thoracic oncology in the UK. A search for non-English language evidence in the peer review literature was beyond the scope of this article; however, many of the concepts, benefits and challenges are likely to be common to all developed countries, particularly those in Europe, and we have tried to include non-British evidence and examples of practice if these were available. We acknowledge, however, that there may be important non-English language evidence on the topic which we have not covered here.

\section{History of multidisciplinary cancer teams}

Some institutions, particularly in the USA, have discussed cancer cases in a multidisciplinary forum since the 1970s. The groups of specialists who met to discuss these cases in the USA made up tumour boards $[1,2]$. Initially these meetings were intended to give specialists the opportunity to keep up to date with new developments relevant to their tumour site, and were an advisory panel which could be consulted by the patient's physician who would consider the opinions offered when deciding how to manage their patient [3]. Tumour boards have now developed into multidisciplinary care teams which, by consensus of opinion, make decisions on diagnosis and management of cancer patients, take responsibility for these decisions and implement them [4]. The team is involved throughout the patient pathway.

Both the US Commission on Cancer and the American College of Surgeons now require health centres to provide evidence of MDT meetings before they can be accredited to deliver cancer care [5]. In the UK, a similar MDT system became a necessity for all hospital trusts in the late 1990s following the publication of government recommendations on care of cancer patients [6]. As a result it was estimated that $96 \%$ of all patients with primary lung cancer were discussed at an MDT meeting in 2011 [7]. The recommendations came in response to poor outcomes for cancer patients, particularly those with lung cancer, and the MDT was to play a role in improving survival and quality of life by improving cancer care and by reducing inequalities in access to treatment.

In addition to the UK and the USA, MDTs are also a standard part of management of thoracic malignancies in Canada [8] and Australia, where they were first introduced approximately 13 years ago [9, 10]. In Spain, as in many other northern European countries, multidisciplinary care was recognised by the government as being a health priority [11, 12]; however, since the Spanish health service was decentralised there is apparently considerable variability in the use of the MDT between hospitals [12, 13]. A European Respiratory Society (ERS) Task Force on lung cancer investigated the organisation of lung cancer care across Europe by surveying lung cancer clinicians in 37 countries. They found MDTs to be a part of lung cancer care in nearly all hospitals included in the survey; however, the proportions of patients discussed and the clinicians who made up the MDT varied considerably. At least one institution in each of Norway, Italy, Finland and Romania reported that they did not discuss any of their patients at an MDT meeting [14]. Similar findings were reported in a Dutch review of care of patients with nonsmall cell lung cancer, which found that in 2004-2005 only two out of six hospitals in the eastern Netherlands had a MDT for lung cancer and the proportion of patients discussed varied between $26 \%$ and $91 \%$ [15].

In North America, Europe and Australia, therefore, thoracic MDTs exist at least in some hospitals with differing degrees of government directives. However, in other parts of the world they are still in the exploratory phase [16].

\section{Who should be in the MDT?}

Recommendations state that membership of the MDT in thoracic oncology should include at least one of each of the following specialists: a pulmonologist with a special interest in thoracic oncology, a radiologist and histopathologist both with thoracic expertise, a clinical nurse specialist, oncologists who can offer both radiotherapy and chemotherapy, a palliative care physician, a thoracic surgeon, and an MDT administrator [17-21]. Regular MDT meetings should be attended by all of these specialists even if they do not hold clinics 
or operate in that hospital. It is common for thoracic surgeons, for example, to be a part of several MDTs each of which meet at different institutions.

In countries with both primary and secondary healthcare systems, the primary care physician plays an important role in co-ordinating patient care, especially at the end of life [22, 23]. Although it is not practical for primary care physicians to attend MDT meetings, because of their wide commitments to many other patients, it is clearly important that there is timely and adequate communication with them. At some institutions, including our own, the primary care physician receives a copy of the MDT recommendations and management plan immediately following the discussion [24].

MDTs need to remain flexible so that they can accommodate advances in diagnosis and treatment. For thoracic oncology this now means a move towards personalised therapies and increasing responsibility for the pathologist and oncologist $[25,26]$.

\section{The MDT meeting}

The MDT meeting or case conference is an integral part of MDT management and all of the core members should attend the regular meetings. This can be a logistical problem due to the number of specialists who need to attend, some of whom attend meetings at more than one hospital. Cases discussed usually include new referrals at the point of diagnosis, but may also include those with newly identified recurrence and other complex cases. In some centres MDT management goes further than a meeting and involves multidisciplinary clinics where the patient could be seen by more than one specialist at the same visit.

MDT meetings may differ in their focus and may include: discussion of all patients at the point of referral; discussions limited to patients with a histological diagnosis, those where the next diagnostic step is not clear, or those for whom it is not appropriate to pursue a tissue diagnosis [27]; discussion of post-operative outcomes, including a review of the surgical resection specimens to provide feedback to the MDT on the treatment decision and an opportunity to decide whether adjuvant therapy should be offered; or rediscussions where clinicians may consult the MDT again if their patient is not fit enough for, or declines, the recommended treatment.

Figure 1 shows an example of a pathway for people with suspected thoracic malignancy including the points at which the MDT could be involved. This pathway is common to many institutions but can invite problems where there is simply too little information about the patient (symptoms, fitness and personal preferences) to make an accurate recommendation. It is, thus, important that the referrer asks the MDT a clear question, rather than expecting a full treatment recommendation. If patients are discussed by the MDT with all of the relevant information available then a decision on treatment options can often be made, subject to the patient's preference [27], although there still may be a decision to do further tests if a specialist feels they are necessary before giving treatment.

If a discussion takes place near to the start of the patient pathway the most appropriate investigations can be arranged immediately and delays to treatment minimised, this is the concept of the "diagnostic MDT" where clinicians work out the best initial approach. In our experience the diagnostic MDT, for reasons of resources and logistics, is usually limited to pulmonologists and radiologists, but it could be argued that other specialists, such as thoracic surgeons, should also contribute to management decisions at this stage.

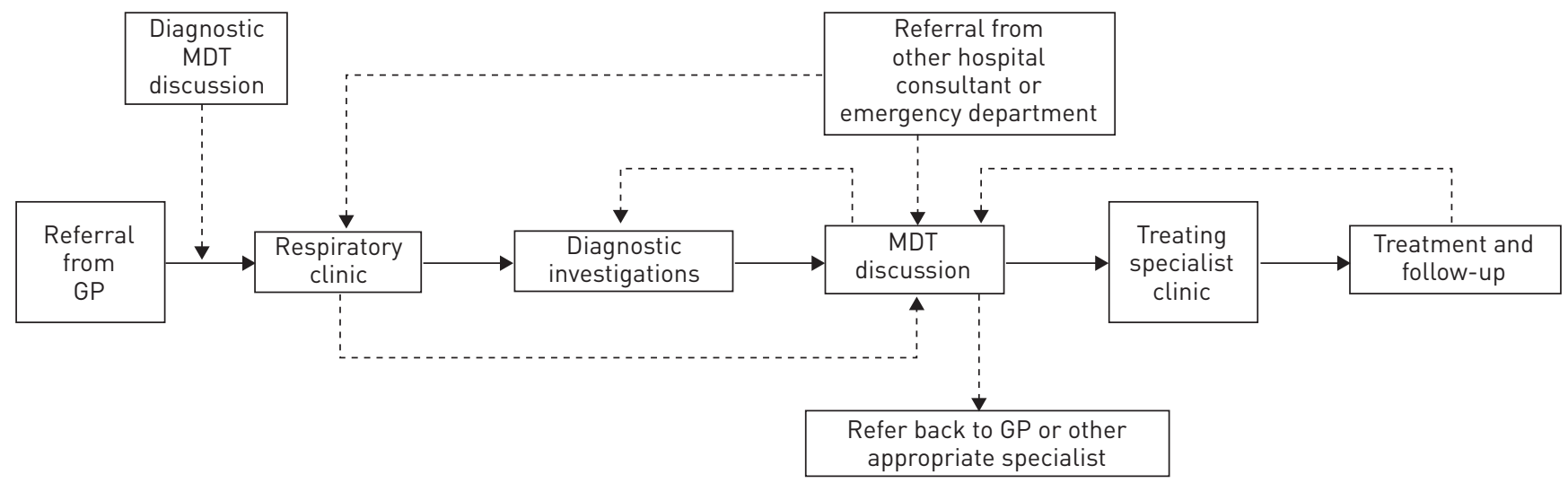

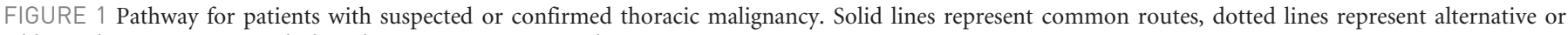
additional routes. MDT: multidisciplinary team; GP: general practitioner. 


\section{The MDT report}

The outcome of the MDT meeting should be recorded and made accessible to all healthcare professionals involved in the patient's care. The importance of timely communication with other healthcare professionals providing care has already been discussed. The nature of MDT reports varies considerably but should ideally include the following: 1) demographics and the results of investigations; 2) naming of stage-dependent guideline recommended treatment; 3) treatment plan and reasons for nonadherence to guideline recommendations; 4) whether the MDT made a unanimous or a majority decision; 5) common treatment side-effects and recommended management for these [24]; and 6) a clear indication of the likely prognosis and follow-up strategy [24].

\section{The evidence for the effectiveness of the MDT}

The lung is one of the only tumour sites for which a systematic review of the evidence for multidisciplinary management has been published [28]. Unfortunately this review demonstrates the paucity of evidence for MDT practice, particularly any evidence of a causative effect on outcomes, such as increased treatment rates or improved survival. It is possible that the apparent difficulties in proving a measurable positive effect of MDT management (which we will discuss below) might have resulted in publication bias with other studies remaining unpublished due to negative results.

There are two main issues to consider when interpreting the available evidence. First, the definition of MDT management varies considerably between studies and so it is not possible to combine the results in a metaanalysis due to this heterogeneity $[28,29]$. A UK study, for example, compared outcomes for 88 patients randomised to either the conventional diagnostic route or a new two-stop centralised pathway, which involved a day case admission to the local tertiary centre as well as early MDT discussion [30]. This pilot study remains the only randomised study, to our knowledge, on the subject. It demonstrated a reduction in delays to diagnosis (but not between diagnosis and treatment) with the centralised pathway, which could be the reason that there were also increased rates of radical treatment and better patient experience. The MDT may reduce the time between diagnosis and treatment because the relevant specialist will be made aware of the need for their input early on in the pathway and so can plan their clinic or procedure lists accordingly, although evidence to support this is scarce $[28,31]$.

The second difficulty with studies that look for improvements in outcomes associated with the introduction of MDT management is the extent of other organisational and treatment changes which have taken place over the same period of time [32]. MDT management of lung cancer was first recommended amid the recognition that survival for this group of patients was extremely poor $[6,17,33,34]$. Improved staging with positron emission tomography (PET)-computed tomography, promotion of symptom awareness and early diagnosis, more funding for treatments such as radiotherapy [17], advances in treatments, particularly targeted therapies [35], and minimally invasive surgical techniques, are all likely to have contributed to any improvements in survival since the introduction of MDT management [36]. One study reported a significant increase in resection rates after the introduction of an MDT meeting in 1997. However, this study was designed to measure the effect of a change in the thoracic surgical service from a cardiothoracic surgeon to a specialist thoracic surgeon [37]. Thus, an independent effect of the introduction of the MDT could not be determined.

\section{Potential benefits of the MDT}

The potential advantages and disadvantages of MDT management are summarised in table 1.

\section{Benefits for the patient}

With the above caveats in mind, there is some evidence that MDTs are beneficial for patients. Several studies have found that MDT management plans show good concordance with evidence based guidelines $[9,11,38]$, and that MDTs increase overall treatment rates, probably through the consideration of more patients with borderline stage or fitness for intervention [37, 39, 40]. Some authors have also reported improvements in survival [39,49], but could not account for confounding by other advances or improvements in the care of patients with thoracic malignancies.

Survival is not the only important factor in thoracic oncology and given the high proportion of patients who present with metastatic disease, for whom treatment options are limited, patient satisfaction and quality of life are extremely important. Boxer et al. [41] retrospectively reviewed the records of 988 lung cancer patients diagnosed in south-west Sydney between 2005 and 2008 using their Clinical Cancer Registry and MDT databases; approximately half were discussed at an MDT meeting and the other half were not. They found the MDT discussion to be an independent predictor of receiving radiotherapy, chemotherapy and referral to palliative care, and concluded that this could potentially translate to an improvement in 
TABLE 1 Advantages and disadvantages of multidisciplinary team (MDT) management for thoracic malignancies

Patients
More likely to receive guideline concordant treatment $[9,11,38]$ More likely to receive any treatment [37-41]

Probable reduction in time from diagnosis to treatment $[30,38]$ Possible improvement in quality of life with more palliative care [41] Protected time for discussion of complex patients Improved communication between MDT members May improve communication with GPs Back-up when management deviates from guidelines Opportunity for education and to keep up to date with new developments

Means of maintaining high standard of service by identifying areas for improvement $[44,45]$ Improved job satisfaction [46, 47]

Facilitates identification of cases and data collection for epidemiological research [7]

Increase number of patients entered into clinical trials [31, 48]

May reduce inequalities in access to treatment Facilitates monitoring of performance [45]
Potential for delays if inadequate information available for discussion

Delays for some patients if initial treatment plan not appropriate [42]

Time required for meetings plus meeting preparation and travel

Potential conflicts of opinion with other members Medico-legal concerns over team-based decisions [43] Potential for deskilling through over-reliance on MDT for decisions

Financial costs

GP: general practitioner.

quality of life, if not survival. While Boxer et al. [41] excluded the effects of time (patients were discussed or not discussed at the discretion of their clinician), this was not a randomised trial and it is possible that those who required treatment were discussed in order to facilitate that referral. There was no evidence of improved survival in people discussed by the MDT despite the increase in treatment rates and the MDT group being significantly younger than those who were not discussed [41]. These findings were corroborated in a large study of almost 25000 patients with lung cancer using the US Veterans Affairs Central Cancer Registry which found that treatment rates for certain groups of patients with lung cancer increased in the presence of a tumour board but that there was no effect on survival [50].

Another US study looked at the effect of a multidisciplinary clinic in addition to the MDT meeting: RIEDEL et al. [31] compared the time from diagnosis to treatment in 244 patients who were seen at an MDT thoracic oncology clinic with 101 patients who were seen in regular clinics after the MDT clinic closed. There were no differences in survival, time to treatment, or treatment rates between the two groups; however, patients were still discussed at a weekly MDT meeting after the clinic was closed.

\section{Benefits for the clinician}

It seems to be quite clear that MDTs help clinicians to follow established guidelines and they also provide an opportunity to streamline decision making and management. One example of this in breast cancer is the use of a computerised decision aid which, on entering patient features such as stage and histology, provides a recommendation for management based on existing guidelines [51]. This has the potential to save time, to reduce errors or omissions in care, and to ensure uniform access to care for all.

The decision not to follow established guidelines may be made by a single clinician on the basis of their knowledge and experience; however, the MDT provides support for these difficult clinical decisions and should the outcome not be favourable may help the clinician to justify their actions to themselves and the patient. In addition the MDT provides an opportunity for people to question management plans, for example the clinical nurse specialist who has an invaluable role as the patient advocate.

Tumour boards were originally developed as educational sessions rather than to directly improve the management of the patient. MDT meetings can also be educational, providing opportunities to view radiology images and pathology slides in the presence of an expert radiologist and histopathologist, and opportunities to discuss new or complex treatments or techniques. A qualitative study from Australia confirmed that MDT members valued the educational benefit of attending team meetings and also reported that the meetings facilitated the inclusion of staff from radiology and pathology into the treatment team, leading to closer working relationships [52].

The MDT meeting also provides an opportunity to review treatment recommendations, particularly after complications or deaths related to treatment, so that the team can learn from these events. One published 
example of this came from an MDT at a UK teaching hospital who reviewed all of their PET-positive resection specimens to see how many proved to be benign. Of the 78 patients with a solitary pulmonary nodule in which a tissue diagnosis was not obtained prior to surgical resection, $8 \%$ had benign histology mostly due to an inflammatory or infective process [44].

In addition to the opportunity for education and self-appraisal and support for decision making, it has also been suggested that the cancer MDT meeting is enjoyed by some clinicians and that their job satisfaction improved as a result $[46,47]$. One of the predominant reasons for this seems to be the feeling that they provide their patients with a better service [47], and the MDT provides a means of monitoring and assessing services at both local and national levels. Important issues surrounding the care of an individual patient may be identified by one member of the team, or audits of MDT practice may be performed, which can be presented to the whole team so that changes to the service can be made with the input and agreement of all team members. At a national level, the MDT can be the vehicle through which the performance of the whole thoracic oncology team is evaluated and improved.

\section{Benefits for the wider population}

The MDT set-up facilitates identification of cases for national databases as well as local audit. The UK National Lung Cancer Audit is one of the most detailed and comprehensive databases of lung cancer in Europe, capturing an estimated $98 \%$ of lung cancer cases annually and collecting data on stage, performance status, histology, treatment and outcome [7]. This provides an excellent resource for epidemiological analysis of lung cancer outcomes, identification of inequalities in care and potential organisational factors which may influence outcomes for patients. Without the MDT structure it is unlikely that the case ascertainment and level of detail would be achievable and research output would suffer. The MDT meeting and subsequent clinic are also a means of identifying and recruiting patients who are suitable for clinical trials $[31,48]$. If these patients were seen in individual clinics it would be much more difficult to identify suitable patients.

\section{Potential problems with MDT management \\ Problems for the patient}

Delays are very distressing for patients and could adversely affect outcomes. While some studies found that MDT management, particularly when combined with a one stop diagnostic clinic, reduced delays between referral and diagnosis, or diagnosis and treatment [30], delays may actually occur as a result of the MDT. In some cases new information comes to light after the MDT meeting (usually as a result of a thorough clinical assessment of patient fitness) meaning that the agreed plan is no longer appropriate. Retrospective analyses have found that between $4 \%$ and $16 \%$ of MDT treatment plans were not actually implemented $[11,53]$, and this may cause delays because of the need to refer to a different specialist [42]. The reasons that MDT recommendations were not implemented were usually patient choice or deterioration in fitness $[11,53,54]$; however, it is possible that last-minute cancellations of procedures or clinic appointments may affect the services available for other patients.

There is variation between institutions in the number of patients discussed at each thoracic MDT meeting. While most high-volume centres offer at least one MDT meeting per week smaller institutions may not have sufficient cases to run a weekly meeting, so patients may wait longer for a management plan. A similar problem arises for rarer tumours such as mesothelioma and Pancoast tumours: clinicians in smaller institutions may not see enough cases to maintain their knowledge and skills in these areas and, therefore, centralisation of care with regional specialist MDTs has been recommended by some $[55,56]$. The centralisation of care for such patients, however, may lead to delays in diagnosis and treatment as they wait for the outcome of the specialist MDT meeting. Furthermore, for rapidly progressive tumours such as small cell lung cancer it may not be appropriate to wait for the MDT meeting before referring to an oncologist. It is, therefore, important to ensure there are systems in place to identify and "fast-track" these patients.

Some MDTs in rural areas serve patients from a large geographic area. This means that patients must be discussed by the MDT and the recommendations communicated to the patient by a different clinician, or that either patients or clinicians travel long distances for consultations.

\section{Problems for the clinician}

The cost implications in terms of clinicians' time are discussed below, but other drawbacks include the potential to increase stress if interactions with colleagues are not amicable [57] and deskilling of clinicians who were previously fully capable of managing patients on their own and may now rely on the MDT to make a decision. While it can be helpful to have the opinions of specialists from all fields within thoracic cancer, there is sometimes a concern regarding medico-legal implications of team-based decisions if a 
patient suffers an adverse outcome after following the MDT-recommended course of treatment, particularly given that not all members contribute to every decision [43].

\section{Problems for the wider population}

It is not clear whether MDT management in thoracic oncology is cost-effective and intuitively it is difficult to argue that it is, given that measures of cost benefits are likely to be in terms of quality-adjusted life-years gained which, as outlined above, is impossible to extract from the benefits of other interventions. It is possible that some unnecessary tests will be avoided as a result of early expert opinion.

Publication of standards documents by governments across the world usually comes with a funding stream for setting up and maintaining MDTs [20]. This reflects the considerable costs involved in the introduction and maintenance of an MDT, including the cost of administration and technology. The need for a regular meeting of the MDT members, which typically takes an hour of each clinician's time every week, is a considerable cost. Some clinicians, who may have limited input into most of the discussions, might legitimately question the cost-effectiveness of their involvement. For example, because most patients have advanced disease, surgeons may feel their time is wasted. It can, however, be argued that surgical expertise is necessary in all patients, including those with stage IV disease, as occasionally there might be an option for surgery in single or oligo-metastatic disease, and it is important that diagnoses of stage IV disease or functional inoperability are agreed by all members to guarantee that management decisions are truly multidisciplinary.

\section{Barriers to effective MDT working}

In 2009 the England and Wales National Cancer Action Team (now part of Public Health England) conducted a survey of over 2000 MDT members for all tumour sites including the lung [58]. The members considered the most important factors for effective MDT working to be those shown in table 2. A qualitative study of urological and gastrointestinal MDT members supports these findings citing nonattendance of key members at meetings, inadequate clinical information and technological failures as barriers to clinical decision making [54].

\section{Attendance at meetings}

MDT members need allocated protected time to attend meetings and it is important that all specialities are represented at each meeting, if not in person then by tele- or videoconferencing [59]. In a survey of attendees at an Australian lung cancer conference in 2010 more than $20 \%$ of the respondents indicated that improvement in attendance at their multidisciplinary meeting was required for palliative care physicians, pathologists and cardiothoracic surgeons [25], and a qualitative study from Spain found that that MDT members did not feel their MDT time was recognised by management as "real work" [12].

\section{Team working and leadership}

The relationships between team members, communication and leadership are all key to ensuring the MDT works effectively. Individual personalities may adversely affect the functioning of an MDT if one or two members make it difficult to hold a reasonable discussion and make a democratic decision. Doctors often dominate the discussions, which may lead to important information about patient preferences being missed [60]. Differences of opinion are, of course, common and not everyone agrees that guidelines are helpful in forming management plans [61]. Good leadership is, therefore, essential in order to ensure the smooth running of an MDT and that decisions are patient-centred and appropriate. Where appropriate differences of opinions about treatment occur, it is important that options are presented to the patient in an unbiased way. When 461 lung cancer doctors in North America were asked their personal preferences for treatment if they were to develop lung cancer, there was no consensus as to their preferred treatment in either of two clinical situations. Specialists from each discipline showed a preference for their own modality of treatment [62]. Typically the MDT is led by a pulmonologist with the aim of excluding these biases; however, the

\section{TABLE 2 Important factors in effective multidisciplinary team working}

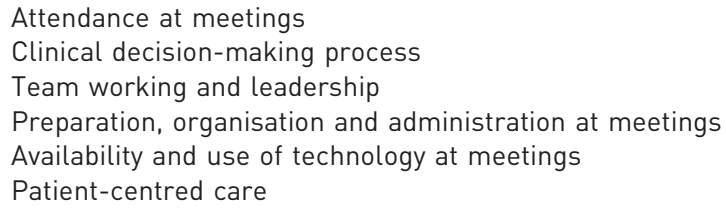


requirements for leadership relate to co-ordination and management of the service, and in some institutions clinical nurse specialists have taken on this role [63].

\section{Preparation, organisation and administration}

Time needs to be allocated to allow for preparation for meetings. Without this, meetings are less efficient. Unfortunately, time allocation for MDT preparation is often insufficient, which can lead to bad preparation and stress for members. In a survey of chest radiologists in the UK, only $50 \%$ had time allocated for meeting preparation [64]. It is also imperative that case notes, reports and images for every patient are available which is why the MDT coordinator role is so important.

Appropriate details about patient fitness and wherever possible the patient's views on treatments must be available so that the MDT recommendations can take this into account [27]. This is particularly important where the patient is elderly or has multiple comorbidities when radical treatments can be risky. Discussions without anyone in the room having met the patient and with scanty information on the referral form may contribute to inappropriate management plans, increase delays to treatment and necessitate repeated discussions at a later date. Inefficiency is further exacerbated by inappropriate referrals to the MDT. Appropriate vetting of such referrals is part of appropriate preparation for the MDT meeting.

\section{Technology}

It is important that technological equipment, such as computer systems and videoconferencing equipment, is available and in working order. Dedicated meeting rooms with adequate facilities for displaying radiographs and pathology slides were described by members as necessary for an effective MDT meeting [58].

\section{Variation and monitoring of quality}

Variation in quality of thoracic MDT management certainly exists [65], but this may be less likely than when a single clinician was responsible for deciding on a management plan. Once an MDT has been established it is important that they monitor their own performance and continually try to improve the service they offer. Starting in 2004, the UK government instituted the National Cancer Peer Review Programme, which is a national quality assurance programme for National Health Service cancer services. The peer review programme involves self-assessments by individual MDTs and external reviews of teams by professional peers, against nationally agreed quality measures [65]. Peer review was part of the National Cancer Reform strategy and was intended to "support quality assurance of cancer services and enable quality improvement" [66].

In 2010 the Improving Lung Cancer Outcomes Project (ILCOP) initiated a process of reciprocal peer review where members of MDTs from different institutions visited neighbouring MDTs and provided feedback on how improvements could be made [45]. This was a popular tool with clinicians as it involved less paperwork and more personal interactions than traditional peer review, and resulted in improvements in quality and efficiency of MDT working at the local level by providing individual recommendations for each institution [67].

\section{Conclusion}

In many European countries, as well as North America and Australia, the MDT is an established mechanism of providing the full range of specialist input for people with thoracic malignancies and is now viewed by most as an essential part of their management. Furthermore, the ERS and American Thoracic Society have recently recommended that all centres offering a thoracic oncology service should have multidisciplinary clinics and a thoracic oncology board [68]. We have discussed advantages and disadvantages of MDT management in detail and these are summarised in table 1 . In terms of the effect on survival and treatment rates for people with thoracic malignancies (which is the reason MDTs were established), the changes seen cannot be attributed to their introduction when other organisational factors are also changing and novel treatments are becoming available to all. The MDT is one way of doing things and far from being just a concept this is now a way of life in some countries.

The majority of the studies cited in this article were performed at institutional or regional level and so we could suggest that large, randomised controlled trials of MDT compared with non-MDT management should be set up, but this is unlikely to happen in countries where MDT management has already been instituted as treatment without them would be seen as a step backwards.

For those countries where cancer MDTs are firmly established the advantages of the MDT are more than intuitive. For individual clinicians there is the convenience of ready and regular access to specialist opinion, including the often levelling effect of alternative views from other specialists, nurses and administrators that make one reflect on management. The convergence of resources can be invaluable to accelerate the progress 
of patients through the pathway. For those in smaller institutions who used to have infrequent contact with other professionals there has been a step-change in access to multidisciplinary management. In addition, the development of MDTs has encouraged greater specialisation of MDT members so that fewer are generalists and more are committed to thoracic oncology.

\section{References}

Gross GE. The role of the tumor board in a community hospital. CA Cancer J Clin 1987; 37: 88-92.

Patkar V, Acosta D, Davidson T, et al. Cancer multidisciplinary team meetings: evidence, challenges, and the role of clinical decision support technology. Int J Breast Cancer 2011; 2011: 831605

3 O’Brien JC. History of tumor site conferences at Baylor University Medical Center. Proc (Bayl Univ Med Cent) 2006; 19: 130-131

4 Fennell ML, Das IP, Clauser S, et al. The organization of multidisciplinary care teams: modeling internal and external influences on cancer care quality. J Natl Cancer Inst Monogr 2010; 2010: 72-80.

5 American College of Surgeons, Commission on Cancer. Cancer Program Standards 2012, Version 1.2: Ensuring Patient-Centered Care. Chicago, American College of Surgeons, 2013. www.facs.org/cancer/coc/programstandards2012. html Date last updated: September 30, 2013. Date last accessed: February 1, 2014.

6 The Expert Advisory Group on Cancer to the Chief Medical Officers of England and Wales. A Policy Framework for Commissioning Cancer Services: A Report by The Expert Advisory Group on Cancer to the Chief Medical Officers of England and Wales. London, Department of Health, 1995.

7 Health and Social Care Information Centre. National Lung Cancer Audit Report 2012. Report for the Audit Period 2011. Leeds, Health and Social Care Information Centre, 2012.

8 Ellis PM. The importance of multidisciplinary team management of patients with non-small-cell lung cancer. Curr Oncol 2012; 19: Suppl. 1, S7-S15.

9 Conron M, Phuah S, Steinfort D, et al. Analysis of multidisciplinary lung cancer practice. Intern Med J 2007; 37: $18-25$.

10 Victorian Government Department of Human Services. Achieving Best Practice Cancer Care. A Guide for Implementing Multidisciplinary Care. Melbourne, Metropolitan Health and Aged Care Services Division, Victorian Government Department of Human Services, 2007.

11 Leo F, Venissac N, Poudenx M, et al. Multidisciplinary management of lung cancer: how to test its efficacy? J Thorac Oncol 2007; 2: 69-72.

12 Prades J, Borràs JM. Multidisciplinary cancer care in Spain, or when the function creates the organ: qualitative interview study. BMC Public Health 2011; 11: 141.

13 Minister of Health and Social Policy. Quality Plan for the National Health System of Spain: Cancer Strategy of the National Health System. Madrid, Minister of Health and Social Policy, 2006.

14 Blum TG, Rich A, Baldwin B, et al. The European initiative for quality management in lung cancer care. Eur Respir J 2014; 43: 1254-1277.

15 Ouwens MMMTJ, Hermens RRPMG, Termeer RAR, et al. Quality of integrated care for patients with nonsmall cell lung cancer. Cancer 2007; 110: 1782-1790.

16 Song P, Wu Q, Huang Y. Multidisciplinary team and team oncology medicine research and development in china. Biosci Trends 2010; 4: 151-160.

17 Department of Health, Clinical Outcomes Group, Cancer Guidance Sub-group. Guidance on Commissioning Cancer Services: Improving Outcomes in Lung Cancer the Manual. London, Department of Health, 1998.

18 Ost DE, Yeung SC, Tanoue LT, et al. Clinical and organizational factors in the initial evaluation of patients with lung cancer: diagnosis and management of lung cancer, 3rd ed. American College of Chest Physicians evidencebased clinical practice guidelines. Chest 2013; 143: Suppl. 5, e121S-e141S.

19 BTS recommendations to respiratory physicians for organising the care of patients with lung cancer. The Lung Cancer Working Party of the British Thoracic Society Standards of Care Committee. Thorax 1998; 53: Suppl. 1, S1-S8.

20 Wright FC, De Vito C, Langer B, et al. Multidisciplinary cancer conferences: a systematic review and development of practice standards. Eur J Cancer 2007; 43: 1002-1010.

21 Brunelli A, Charloux A, Bolliger CT, et al. ERS/ESTS clinical guidelines on fitness for radical therapy in lung cancer patients (surgery and chemo-radiotherapy). Eur Respir J 2009; 34: 17-41.

22 Grunfeld E. Primary care physicians and oncologists are players on the same team. J Clin Oncol 2008; 26: 2246-2247.

23 Bellamy D, Peake M, Williams A. The role of primary care as part of the multidisciplinary team (MDT) in the management of lung cancer: the "dream MDT" report - new guidance from the UK Lung Cancer Coalition. Prim Care Respir J 2013; 22: 3-4.

24 Penel N, Valentin F, Giscard S, et al. General practitioners assessment of a structured report on medical decision making by a regional multidisciplinary cancer committee. Bull Cancer 2007; 94: E23-E26.

25 Bydder S, Hasani A, Broderick C, et al. Lung cancer multidisciplinary team meetings: a survey of participants at a national conference. J Med Imaging Radiat Oncol 2010; 54: 146-151.

26 Davidson MR, Gazdar AF, Clarke BE. The pivotal role of pathology in the management of lung cancer. J Thorac Dis 2013; 5: Suppl. 5, S463-S478.

27 Sharma RA, Shah K, Glatstein E. Multidisciplinary team meetings: what does the future hold for the flies raised in Wittgenstein's bottle? Lancet Oncol 2009; 10: 98-99.

28 Coory M, Gkolia P, Yang IA, et al. Systematic review of multidisciplinary teams in the management of lung cancer. Lung Cancer 2008; 60: 14-21.

29 Hong NJL, Wright FC, Gagliardi AR, et al. Examining the potential relationship between multidisciplinary cancer care and patient survival: an international literature review. J Surg Oncol 2010; 102: 125-134. 
30 Murray PV, O'Brien MER, Sayer R, et al. The pathway study: results of a pilot feasibility study in patients suspected of having lung carcinoma investigated in a conventional chest clinic setting compared to a centralised two-stop pathway. Lung Cancer 2003; 42: 283-290.

31 Riedel RF, Wang X, McCormack M, et al. Impact of a multidisciplinary thoracic oncology clinic on the timeliness of care. J Thorac Oncol 2006; 1: 692-696.

32 Erridge SC, Murray B, Price A, et al. Improved treatment and survival for lung cancer patients in south-east Scotland. J Thorac Oncol 2008; 3: 491-498.

33 Berrino F, Sant M, Verdecchia A, et al. Survival of Cancer Patients in Europe. The EUROCARE Study. Geneva, IARC Scientific Publications, 1995.

34 Fry WA, Phillips JL, Menck HR. Ten-year survey of lung cancer treatment and survival in hospitals in the United States: a national cancer data base report. Cancer 1999; 86: 1867-1876.

35 Blackhall F, Thatcher N, Booton R, et al. The impact on the multidisciplinary team of molecular profiling for personalized therapy in non-small cell lung cancer. Lung Cancer 2013; 79: 101-103.

Spiro SG, Silvestri GA. One hundred years of lung cancer. Am J Respir Crit Care Med 2005; 172: 523-529.

37 Martin-Ucar AE, Waller DA, Atkins JL, et al. The beneficial effects of specialist thoracic surgery on the resection rate for non-small-cell lung cancer. Lung Cancer 2004; 46: 227-232.

38 Freeman RK, Van Woerkom JM, Vyverberg A, et al. The effect of a multidisciplinary thoracic malignancy conference on the treatment of patients with lung cancer. Eur J Cardiothorac Surg 2010; 38: 1-5.

39 Forrest LM, McMillan DC, McArdle CS, et al. An evaluation of the impact of a multidisciplinary team, in a single centre, on treatment and survival in patients with inoperable non-small-cell lung cancer. Br J Cancer 2005; 93: 977-978.

40 Wang J, Kuo YF, Freeman J, et al. Increasing access to medical oncology consultation in older patients with stage IIIIIa non-small-cell lung cancer. Med Oncol 2008; 25: 125-132.

41 Boxer MM, Vinod SK, Shafiq J, et al. Do multidisciplinary team meetings make a difference in the management of lung cancer? Cancer 2011; 117: 5112-5120.

42 Osarogiagbon RU, Phelps G, McFarlane J, et al. Causes and consequences of deviation from multidisciplinary care in thoracic oncology. J Thorac Oncol 2011; 6: 510-516.

43 Evans AC, Zorbas HM, Keaney MA, et al. Medicolegal implications of a multidisciplinary approach to cancer care: consensus recommendations from a national workshop. Med J Aust 2008; 188: 401-404.

44 Clifton IJ, Scarsbrook AF, Muers MF. No lung cancer at resection of PET positive lesions: an audit of the lung cancer multidisciplinary team. Eur Respir J 2009; 33: 941-942.

45 Aveling EL, Martin G, Jiménez García S, et al. Reciprocal peer review for quality improvement: an ethnographic case study of the Improving Lung Cancer Outcomes Project. BMJ Qual Saf 2012; 21: 1034-1041.

46 Taylor C, Graham J, Potts HW, et al. Changes in mental health of UK hospital consultants since the mid-1990s. Lancet 2005; 366: 742-744.

47 Taylor C, Sippitt JM, Collins G, et al. A pre-post test evaluation of the impact of the PELICAN MDT-TME Development Programme on the working lives of colorectal cancer team members. BMC Health Serv Res 2010; 10: 187.

48 McNair AGK, Choh CTP, Metcalfe C, et al. Maximising recruitment into randomised controlled trials: the role of multidisciplinary cancer teams. Eur J Cancer 2008; 44: 2623-2626.

49 Bydder S, Nowak A, Marion K, et al. The impact of case discussion at a multidisciplinary team meeting on the treatment and survival of patients with inoperable non-small cell lung cancer. Intern Med J 2009; 39: 838-841.

50 Keating NL, Landrum MB, Lamont EB, et al. Tumor boards and the quality of cancer care. J Natl Cancer Inst 2013; 105: 113-121.

51 Patkar V, Acosta D, Davidson T, et al. Using computerised decision support to improve compliance of cancer multidisciplinary meetings with evidence-based guidance. BMJ Open 2012; 2: e000439.

52 Devitt B, Philip J, McLachlan SA. Team dynamics, decision making, and attitudes toward multidisciplinary cancer meetings: health professionals' perspectives. J Oncol Pract 2010; 6: e17-e20.

53 Storrar W, Laws D. Effectiveness of decision making at lung cancer multidisciplinary team meetings. Lung Cancer 2011; 71: Suppl. 1, S20.

54 Jalil R, Ahmed M, Green JSA, et al. Factors that can make an impact on decision-making and decision implementation in cancer multidisciplinary teams: an interview study of the provider perspective. Int J Surg 2013; 11: 389-394.

55 Postmus PE. Longkanker: centraliseren voor multidisciplinaire behandeling [Lung cancer: centralisation of multidisciplinary treatment]. Ned Tijdschr Geneeskd 2007; 151: 1382-1384.

56 British Thoracic Society Standards of Care Committee. BTS statement on malignant mesothelioma in the UK, 2007. Thorax 2007; 62: Suppl. 2, iil-ii19.

57 Coates A. Poisons and handwork: interactions between surgeons and medical oncologists. ANZ J Surg 2002; 72: $174-176$.

58 Taylor C, Ramirez A. Multidisciplinary Team Members' Views About MDT Working: Results from a Survey Commissioned by the National Cancer Action Team. London, National Cancer Action Team, 2009.

59 Gagliardi A, Smith A, Goel V, et al. Feasibility study of multidisciplinary oncology rounds by videoconference for surgeons in remote locales. BMC Med Inform Decis Mak 2003; 3: 7.

60 Rowlands S, Callen J. A qualitative analysis of communication between members of a hospital-based multidisciplinary lung cancer team. Eur J Cancer Care 2013; 22: 20-31.

61 Stinchcombe TE, Detterbeck FC, Lin L, et al. Beliefs among physicians in the diagnostic and therapeutic approach to non-small cell lung cancer. J Thorac Oncol 2007; 2: 819-826.

62 Palmer MJ, O'Sullivan B, Steele R, et al. Controversies in the management of non-small cell lung-cancer: the results of an expert surrogate study. Radiother Oncol 1990; 19: 17-28.

63 Snow A, Gallini A. Taking the lead and extending the role of lung cancer clinical nurse specialist. Cancer Nursing Practice 2006; 5: 33-36.

64 Aujayeb A, Shariff A, Talbot L, et al. UK national survey on radiologists' perspective of lung cancer MDT: can we do better? Thorax 2009; 64: A146. 
65 National Cancer Action Team. National Cancer Peer Review Programme Report 2010/2011. An overview of the findings from the 2010/2011 National Cancer Peer Review of Cancer Services in England. London, National Cancer Action Team, 2011.

66 Department of Health. Cancer Reform Strategy. London, Department of Health, 2007.

67 The Health Foundation. Improving Lung Cancer Outcomes Project (ILCOP). Final Report for Award Holders Closing the Gap through Clinical Communities. London, The Health Foundation, 2013.

68 Gaga M, Powell CA, Schraufnagel DE, et al. An official American Thoracic Society/European Respiratory Society statement: the role of the pulmonologist in the diagnosis and management of lung cancer. Am J Respir Crit Care Med 2013; 188: 503-507. 